\title{
Non-linear Stark Effect and Molecular Localization
}

\author{
Vincenzo Grecchi ${ }^{1}$, André Martinez ${ }^{2}$ \\ 1 Università di Bologna, Dipartimento di Matematica, I-40127 Bologna, Italy \\ 2 Université de Paris-Nord, Département de Mathématiques (CNRS, URA 742), Av. J. B. Clément, \\ F-93430 Villetaneuse, France
}

Received: 7 July 1993/in revised form: 26 January 1994

\begin{abstract}
We consider a non-linear Stark effect as a model for localization and symmetry breaking of a molecule in a gas. By a comparison method with respect to the linear Stark effect, we prove the existence of level bifurcation and symmetry breaking at a critical value of the gas pressure exponentially small for large nuclear masses. Extending the Davies results, we confirm the predictions of Claverie-Jona Lasinio for pyramidal molecules as the ammonia one.
\end{abstract}

\section{Introduction}

The empirical chemical models for molecules imply certain asymmetries and structures in apparent contradiction with quantum mechanical principles. Even the accepted features of the water molecule [Pr] are not proved to be in agreement with straightforward application of quantum mechanics. One possible explanation of this phenomenon lies on the existence of non-stationary states that are more stable than the stationary ones to the environment interactions.

As a particularly interesting example of molecule with structure we have the ammonia one $\mathrm{NH}_{3}$ (or $\mathrm{AsH}_{3}, \mathrm{PH}_{3}$ ). To be more clear, following Wightman [Wi], it is better to consider the similar molecule NHDT (or AsHDT, PHDT) in which the three hydrogen nuclei are distinguished by isotopes. Such molecules have a pyramidal structure with the HDT in a triangular basis and the $\mathrm{N}$ in the vertex. Actually there are two kinds of such pyramides with different chirality. Chiral molecules can be discovered by the "optical activity": they are able to rotate the polarization plane of light. Quantum mechanics predicts symmetrical molecules with the $\mathrm{N}$ nucleus delocalized in both possible vertices. In the Born-Oppenheimer approximation the $\mathrm{N}$ nucleus feels a double well potential as it is clear since 1927 [Ha]. Quantum levels are split in two because of the two possible even/odd inversion states. States initially localized in one well (chiral states) oscillate (beating effect) from one well to the other. Physically we always observe stable (without oscillations) chiral molecules in a gas at a pressure large enough (at fixed temperature). All the models used for 
explaining the phenomenon consider the electric interaction of the molecule with the environment [Ma, An, Pf, ClJo1]. In particular Claverie and Jona Lasinio [ClJo1] consider the dipole electric field of an asymmetric molecule polarizing a dielectric medium. The reaction field stabilizes the asymmetric state of the molecule. In this way the asymmetric state of the molecule becomes a stationary state of a non-linear Schrödinger equation of the Stark type, for a large enough pressure. This model was discussed in [ClJo1] with quantitative comparison with experimental data. The same model can be applied to the electron state in $\mathrm{H}_{2}^{+}$in order to explain the instability for large nuclear distance by localization of the electron near one nucleus [ClJo2].

We consider a class of models of the kind suggested in [ClJo1] (see also [Pr] for an explicit model in the atomic case) with Hamiltonian

$$
\psi \mapsto H_{\tau}(\psi)=P_{0} \psi+\tau\langle\psi, W \psi\rangle . W \psi,
$$

where $P_{0}$ is invariant under a symmetry $S$ and has a pair of isolated eigenvalues with symmetric/antisymmetric eigenfunctions, $W=\left(W_{1}, \ldots, W_{n}\right)$ is a bounded vectorvalued self-adjoint operator with $S W=-W S$, and $\tau$ is a real parameter behaving as the opposite of the square of the pressure. We compare the eigenvalues of $H_{\tau}$ with those of the linear operator $P_{\nu}=P_{0}+\nu . W\left(\nu \in \mathbb{R}^{n}\right)$. In the case of the pyramidal molecule, $\nu . W$ is the potential of a locally uniform electric field, so that $H_{\tau}$ is a non-linear Stark operator. Then we are able to prove the existence of a spontaneous symmetry breaking (as $\tau$ varies) by bifurcation of the symmetric state, and the appearance of a degenerate ground state. We also give the critical parameters at the bifurcation point, the latter being of the same order as the splitting and so exponentially small for large nuclear masses. The picture is similar to the usual Stark effect if we consider the parameter $\tau$ in place of the field strength, with some differences discussed below.

Let us notice that Wightman and Glance [WiGl, Wi] consider such models relevant for the discussion on superselection rules and deserving complete rigorous study. Before the Claverie-Jona Lasinio papers, Davies [Da] proposed some models of molecular localization with further justifications, and gave some rigorous results that we extend here. In particular Davies proved the existence of symmetry breaking for large nuclear masses, and here we make precise the statement of [ClJo1] that under semiclassical conditions, the localization is given by a perturbation of the same order of magnitude as the splitting.

The paper is organised in the following way: In the next section we state the main result, in Sect. 2 we consider the linear Stark problem, and in Sect. 3 we give the behavior of the eigenvalues and eigenvectors in the linear case. In Sect. 4 we prove the main result on symmetry breaking.

\section{Notations and Main Result}

Let $P_{0}$ be a self-adjoint operator on a Hilbert space $\mathscr{H}$, with domain $\mathscr{H}_{0}$, and let $S$ be a (unitary) symmetry on $\mathscr{H}$ satisfying:

$$
S\left(\mathscr{H}_{0}\right) \subset \mathscr{H}_{0} \text { and } P_{0} S=S P_{0} \quad \text { on } \mathscr{H}_{0} \text {. }
$$

We assume that $P_{0}$ admits two isolated, successive, simple eigenvalues $E^{ \pm}=E_{0} \mp \mu$ (with $\mu>0$ ), associated to normalized eigenvectors $\varphi_{0}^{+}$and $\varphi_{0}^{-}$which satisfy the following parity conditions:

$$
S \varphi_{0}^{ \pm}= \pm \varphi_{0}^{ \pm}
$$


Denote

$$
\lambda=\frac{1}{2} \operatorname{dist}\left(\left\{E^{+} ; E^{-}\right\} ; \sigma\left(P_{0}\right) \backslash\left\{E^{+}, E^{-}\right\}\right) \in \mathbb{R}_{+}^{*} .
$$

For some fixed $n \in \mathbb{N}^{*}$ let also $W_{1}, \ldots, W_{n}$ be $n$ bounded self-adjoint operators on $\mathscr{H}$, which anticommute with $S$ :

$$
W_{\jmath} S=-S W_{\jmath} \quad(j=1, \ldots, n)
$$

and set $W=\left(W_{1}, \ldots, W_{n}\right)$. For $\tau \in \mathbb{R}$, we consider the following family of nonlinear Schrödinger operators acting on $\mathscr{H}_{0}$ :

$$
H_{\tau}(\psi)=P_{0} \psi+\tau\langle W \psi, \psi\rangle . W \psi
$$

and we investigate the solutions $(E, \psi) \in\left(E^{+}-\lambda, E^{-}+\lambda\right) \times \mathscr{H}_{0}$ of the spectral problem:

$$
\left\{\begin{array}{l}
H_{\tau}(\psi)=E \psi \\
\|\psi\|_{\mathscr{B}}=1
\end{array}\right.
$$

Denoting

$$
\varrho_{0}=\left\langle W \varphi_{0}^{+}, \varphi_{0}^{-}\right\rangle \in \mathbb{C}^{n},
$$

we assume that $\varrho_{0} \neq 0$ and that the choice of $\varphi_{0}^{ \pm}$can be made in such a way that

$$
\varrho_{0} \in \mathbb{R}^{n} \backslash\{0\} .
$$

Notice that when $\mathscr{H}$ is a $L^{2}$-space and $P_{0}$ commutes with conjugation, $\varphi_{0}^{ \pm}$can always be taken real. In this case, $W$ locally represents the position operator. Also, $\varrho_{0}$ can be seen as a mean value of $W$, since we have $\varrho_{0}=\left\langle W \psi_{0}, \psi_{0}\right\rangle$ where $\psi_{0}=\left(\varphi_{0}^{+}+\varphi_{0}^{-}\right) / \sqrt{2}$ is in some sense a one-well localized state.

We also denote

$$
\lambda_{1}=\mu+\lambda
$$

and for $\tau_{0} \in \mathbb{R}^{*}$, we define:

$$
\eta=\eta\left(\lambda, \mu, \varrho_{0},\|W\|, \tau_{0}\right)=\frac{\tau_{0}\|W\|^{2}}{\lambda}\left(1+\frac{\|W\|}{\left|\varrho_{0}\right|}+\frac{\|W\|^{2}}{\varrho_{0}^{2}}+\frac{\lambda_{1}}{\lambda}\right)+\frac{\mu \lambda_{1}\|W\|^{2}}{\lambda^{2} \varrho_{0}^{2}} .
$$

Notice that $\eta$ is dimensionless when $\tau_{0}\|W\|^{2}$ has the dimension of an energy. Then our result is:

Theorem 1.1. Under assumptions (1.1)-(1.4) and (1.7), there exist universal constants $C_{0}$ and $\eta_{0}$ such that if $\frac{\mu}{\tau_{0} \varrho_{0}^{2}}+\eta\left(\lambda, \mu, \varrho_{0},\|W\|, \tau_{0}\right) \leq \eta_{0}$, then there exist $\left.\tau_{ \pm} \in\right]-\tau_{0}, \tau_{0}[$ satisfying:

$$
\left|\tau_{ \pm} \mp \frac{\mu}{\varrho_{0}^{2}}\right| \leq C_{0} \eta \frac{\mu}{\varrho_{0}^{2}}
$$

and such that:

(i) If $\tau \in\left[\tau_{-}, \tau_{+}\right]$, the only solutions of (1.6) are the symmetrical ones: $\psi=\varphi_{0}^{ \pm}$, $E=E^{ \pm}$.

(ii) If $\tau \in\left[-\tau_{0}, \tau_{-}[\right.$, then (1.6) admits exactly 4 solutions. Two of them are the symmetrical ones as in (i), and the two other ones $\tilde{\varphi}_{\tau}^{-}$and $S \tilde{\varphi}_{\tau}^{-}$are both associated to the same energy $E_{\tau}^{-}$which satisfies:

$$
\left|E_{\tau}^{-}-\left(E_{0}+\tau \varrho_{0}^{2}\right)\right| \leq C_{0} \eta|\tau| \varrho_{0}^{2} .
$$


(iii) If $\left.\tau \in] \tau_{+}, \tau_{0}\right]$, the result is analogous to (ii), with states $\tilde{\varphi}_{\tau}^{+}$and $S \tilde{\varphi}_{\tau}^{+}$associated to $E_{\tau}^{+}$satisfying:

$$
\left|E_{\tau}^{+}-\left(E_{0}+\tau \varrho_{0}^{2}\right)\right| \leq C_{0} \eta|\tau| \varrho_{0}^{2} .
$$

Moreover, the states $\tilde{\varphi}_{\tau}^{ \pm}$appearing in (ii) and (iii) satisfy:

$$
\left|\left\langle W \tilde{\varphi}_{\tau}^{ \pm}, \tilde{\varphi}_{\tau}^{ \pm}\right\rangle \mp \frac{\sqrt{\tau^{2}-\tau_{ \pm}^{2}}}{|\tau|} \varrho_{0}\right| \leq C_{0} \eta\left|\varrho_{0}\right| \frac{\sqrt{\tau^{2}-\tau_{ \pm}^{2}}}{|\tau|} .
$$

Remark 1.2. In other words, our result says that only for $\varrho_{0}^{2}|\tau|>\varrho_{0}^{2}\left|\tau_{ \pm}\right| \approx \mu$ (i.e. for the modulus of the mean value of the perturbing potential greater than one half of the splitting, a condition essentially in agreement with experimental data: see [ClJo1]), there exist localized states associated to non-linear eigenvalues $E_{\tau}^{ \pm} \approx E_{0}+\tau \varrho_{0}^{2}$. Such eigenvalues should be compared to those of the usual Stark effect operator: $P_{0}+\tau \varrho_{0} . W$. On the other side, in the region $|\tau| \leq\left|\tau_{ \pm}\right|$we find constant eigenvalues $E^{ \pm}$. In some sense, the well known behaviour of the Stark effect eigenvalues (i.e. linear for large $\tau$ and quadratic for small $\tau$ ) is replaced here by a "close" polygonal line.

The last statement of the theorem means in particular that the dipole moment of the localized states has a square root behaviour near the critical point.

Remark 1.3. Actually, for the specific physical problem we are discussing here, only non-positive $\tau$ is of interest. So that the perturbation is negative and makes the lower eigenvalue bifurcate, yielding a new degenerate $\tau$-depending lower eigenvalue. We also consider positive values of $\tau$ for a better understanding of the model. In this latter case the upper eigenvalue bifurcates, yielding a new $\tau$-depending upper eigenvalue. Both the $\tau$-dependent localized states are on the same line, having the slope $\varrho_{0}^{2}$ and reaching $E_{0}$ (the one well eigenvalue) when continued up to $\tau=0$.

Application to the Semiclassical Case. Assume

$$
P_{0}=-h^{2} \Delta+V(x)
$$

where $h^{2}=1 / 2 M$ ( $M$ representing the mass of the nuclei), and $V$ is symmetric with respect to the hyperplane $\left\{x_{n}=0\right\}$ of $\mathbb{R}^{n}$ and admits two non-degenerate point-wells at energy $0=\operatorname{Min} V$. Consider $P_{0}$ as acting on $L^{2}(\Omega)$ with Dirichlet boundary condition, where $\Omega$ is a connected open set containing the wells, and take $W(x)=x_{n}$. Then the first eigenvalues of $P_{0}$ are equivalent to some $e_{\jmath} h(j=1,2, \ldots)$ as $h$ tends to 0 (see e.g. [Si, HeSj]). Considering only the first two eigenvalues of $P_{0}$ (which are both asymptotically equivalent to $\lambda_{1}(h)=e_{1} h+O\left(h^{3 h}\right)$ ), the conditions of Theorem 1.1 are satisfied in that case for $h$ small enough if we take:

$$
\tau_{0}=\frac{1}{C} h
$$

with some constant $C>0$ sufficiently large (but independently of $h$ ). We also see that the critical parameters $\left|\tau_{ \pm}\right|$are of the same order of magnitude as the splitting between the first two eigenvalues of $P_{0}$, i.e. of order $e^{-S_{0} / h}$ for some constant $S_{0}>0$. In particular, taking $\tau=\tau(h)<0$ with $e^{S_{0} / h}|\tau(h)| \rightarrow+\infty$ and $\tau(h) / h \rightarrow 0$ as $h \rightarrow 0$, we find that the (degenerate) ground state $\left(E, \psi^{ \pm}\right)$of the non-linear system (1.6) satisfies in this case:

$$
\begin{gathered}
E=\lambda_{1}(h)-|\tau(h)| \varrho_{0}^{2}(1+o(1)), \\
\left\langle W \psi^{ \pm}, \psi^{ \pm}\right\rangle= \pm \varrho_{0}(1+o(1)),
\end{gathered}
$$

as $h$ tends to 0 . 
Similarly, our result can also be applied to the molecular Schrödinger operator in the Born-Oppenheimer approximation (see [KMSW]). In this case, the well is invariant under the action of $O(3)$, and one can take $W=x$ were $x$ denotes the coordinates of the nuclei.

\section{Linear Preliminaries}

By adding a constant to $P_{0}$, we assume from now on that $E_{0}=0$.

In this section, we investigate the spectral properties of the families of operators:

$$
P_{\nu}=P_{0}+\nu \cdot W=P_{0}+\sum_{j=1}^{n} \nu_{\jmath} W_{\jmath}
$$

when $\nu=\left(\nu_{1}, \ldots, \nu_{n}\right) \in \mathbb{R}^{n}$ is small, and near the energies $E^{ \pm}$. With $\lambda$ defined as in (1.3), let $\gamma$ be a complex simple loop encircling $E^{+}$and $E^{-}$with the rest of $\sigma\left(P_{0}\right)$ in its exterior, and with:

$$
\operatorname{dist}\left(\gamma ; \sigma\left(P_{0}\right)\right)=\lambda .
$$

Then, the operator:

$$
\Pi_{0}=\frac{1}{2 i \pi} \int_{\gamma}\left(z-P_{0}\right)^{-1} d z
$$

is the spectral projector of $P_{0}$ associated to $E^{-}$and $E^{+}$. For $\nu$ satisfying

$$
\sum_{1}^{n}\left|\nu_{\jmath}\right| \cdot\left\|W_{\jmath}\right\|<\lambda
$$

we can also set:

$$
\Pi_{\nu}=\frac{1}{2 i \pi} \int_{\gamma}\left(z-P_{\nu}\right)^{-1} d z=\frac{1}{2 i \pi} \sum_{k \geq 0} \nu^{k} \int_{\gamma}\left[\left(z-P_{0}\right)^{-1} W\right]^{k}\left(z-P_{0}\right)^{-1} d z
$$

which is a spectral projector of $P_{\nu}$, its range being generated by

$$
\left\{\begin{array}{l}
\varphi_{\nu}^{+}=\Pi_{\nu} \varphi_{0}^{+} \\
\varphi_{\nu}^{-}=\Pi_{\nu} \varphi_{0}^{-}
\end{array}\right.
$$

Our aim is to compute rather precisely the eigenvectors and eigenvalues of $P_{\nu} \Pi_{\nu}$ for $\nu$ small enough. We first consider the matrix:

$$
A_{\nu}=\left(\begin{array}{cc}
\left\langle\varphi_{\nu}^{+}, \varphi_{\nu}^{+}\right\rangle & \left\langle\varphi_{\nu}^{+}, \varphi_{\nu}^{-}\right\rangle \\
\left\langle\varphi_{\nu}^{-}, \varphi_{\nu}^{+}\right\rangle & \left\langle\varphi_{\nu}^{-}, \varphi_{\nu}^{-}\right\rangle
\end{array}\right)
$$

where $\langle.,$.$\rangle stands for the \mathscr{H}$-bracket.

Introducing the parameters:

$$
\begin{aligned}
& \varepsilon_{1}=\frac{1}{\lambda} \sum_{j=1}^{n}\left|\nu_{j}\right| .\left\|W_{j}\right\|, \\
& \varepsilon_{2}=\frac{\pi \lambda+2 \mu}{\pi \lambda} \frac{\varepsilon_{1}}{1-\varepsilon_{1}},
\end{aligned}
$$

we then have: 
Lemma 2.1. For $\operatorname{Max}\left\{\varepsilon_{1}, \varepsilon_{2}\right\}<1, A_{\nu}$ is invertible and we can write:

$$
A_{\nu}^{-1 / 2}=\left(\begin{array}{cc}
1+\alpha_{1} & \alpha_{2} \\
\bar{\alpha}_{2} & 1+\alpha_{3}
\end{array}\right)
$$

where the $\alpha_{j}$ 's are analytic functions of $\nu$ satisfying:

$$
\left\{\begin{array}{l}
\alpha_{j}(0)=0 \quad(j=1,2,3) ; \\
\alpha_{1} \text { and } \alpha_{3} \text { are even } ; \\
\alpha_{2} \text { is odd }
\end{array}\right.
$$

Moreover, for any $\delta>0$ there exists $C_{\delta}$ depending only on $\delta$ such that:

$$
\begin{aligned}
& \left|\alpha_{1}\right|+\left|\alpha_{3}\right| \leq C_{\delta} \varepsilon_{2}^{2}, \\
& \left|\alpha_{2}\right| \leq C_{\delta} \varepsilon_{2},
\end{aligned}
$$

for all $\varepsilon_{2} \in[0,1-\delta]$.

Proof. In view of (2.5) and the fact that we can take length $(\gamma)=2 \pi \lambda+4 \mu$ in (2.5), we see that if $\varepsilon_{1}<1$,

$$
\left\|A_{\nu}-1\right\| \leq \varepsilon_{2}
$$

and therefore $A_{\nu}$ is invertible for $\varepsilon_{2}<1$, and its inverse depends holomorphically on $\nu$. Moreover, since

$$
P_{-\nu}=S P_{\nu} S
$$

we get by (1.2):

$$
\left\{\begin{array}{l}
\varphi_{-\nu}^{+}=S \varphi_{\nu}^{+} \\
\varphi_{-\nu}^{-}=-S \varphi_{\nu}^{-} .
\end{array}\right.
$$

Using the fact that $S$ is unitary, we deduce from (2.11) that $A_{\nu}$ has the form:

$$
A_{\nu}=\left(\begin{array}{ll}
f_{1}(\nu) & f_{2}(\nu) \\
f_{2}(\nu) & f_{3}(\nu)
\end{array}\right),
$$

where $f_{1}$ and $f_{3}$ are even, and $f_{2}$ is odd. Then it is easy to verify that such a form is invariant under the inversion and the square root of $2 \times 2$ matrices. Finally, the estimates on the coefficients $\alpha_{j}$ 's follow from (2.9).

Now, define:

$$
\left(u_{\nu}^{+}, u_{\nu}^{-}\right)=\left(\varphi_{\nu}^{+}, \varphi_{\nu}^{-}\right) A_{\nu}^{-1 / 2}
$$

which forms an orthonormal basis of $\operatorname{Ran} \Pi_{\nu}$.

Then, the main result of this section is:

Proposition 2.2. Under assumptions (1.1)-(1.4), and as long as $\operatorname{Max}\left\{\varepsilon_{2}, \varepsilon_{2}\right\}<1$, the matrix of $P_{\nu} \Pi_{\nu}$ in the basis $\left(u_{\nu}^{+}, u_{\nu}^{-}\right)$can be written:

$$
\mathscr{C}_{\nu}=\left(\begin{array}{ll}
\frac{E^{+}+\beta_{1}(\nu)}{\varrho_{0} \cdot \nu+\beta_{2}(\nu)} & \varrho_{0} \cdot \nu+\beta_{2}(\nu) \\
E^{-}+\beta_{3}(\nu)
\end{array}\right),
$$

where the $\beta_{j}$ 's are analytic functions of $\nu, \beta_{1}$ and $\beta_{3}$ are even, $\beta_{2}$ is odd, and for any $\delta>0$ there exists $C_{\delta}>0$ depending only on $\delta$ such that:

$$
\begin{aligned}
& \left|\beta_{1}\right|+\left|\beta_{3}\right| \leq C_{\delta} \lambda_{1} \varepsilon_{2}^{2}, \\
& \left|\beta_{2}\right| \leq C_{\delta} \lambda_{1} \varepsilon_{2}^{3}
\end{aligned}
$$

for $0 \leq \varepsilon_{2} \leq 1-\delta$. 
Proof. By (1.9), we have:

$$
\Pi_{\nu}=\Pi_{0}+\sum_{k=1}^{+\infty} q_{k}(\nu)
$$

with:

$$
\begin{aligned}
q_{k}(-\nu) & =(-1)^{k} q_{k}(\nu), \\
\left\|q_{k}\right\| & \leq \frac{\pi \lambda+2 \mu}{\mu \lambda} \varepsilon_{1}^{k} .
\end{aligned}
$$

Then, decomposing $\varphi_{\nu}^{ \pm}$according to (2.13), and noticing that $\left\langle P_{\nu} \varphi_{\nu}^{ \pm}, \varphi_{\nu}^{ \pm}\right\rangle$is even by (2.10)-(2.11), we get:

$$
\begin{aligned}
\left\langle P_{\nu} \varphi_{\nu}^{ \pm}, \varphi_{\nu}^{ \pm}\right\rangle & =\left\langle P_{\nu} \varphi_{0}^{ \pm}, \varphi_{\nu}^{ \pm}\right\rangle \\
& =E^{ \pm}+\sum_{k=1}^{+\infty}\left(\left\langle P_{0} \varphi_{0}^{ \pm}, q_{2 k}(\nu) \varphi_{0}^{ \pm}\right\rangle+\left\langle\nu . W \varphi_{0}^{ \pm}, q_{2 k-1}(\nu) \varphi_{0}^{ \pm}\right\rangle\right)
\end{aligned}
$$

Similarly, and also making an easy residue-calculation, we find:

$$
\left\langle P_{\nu} \varphi_{\nu}^{+}, \varphi_{\nu}^{-}\right\rangle=\varrho_{0} . \nu+\sum_{k=1}^{+\infty}\left(\left\langle P_{0} \varphi_{0}^{+}, q_{2 k+1}(\nu) \varphi_{0}^{-}\right\rangle+\left\langle\nu . W \varphi_{0}^{+}, q_{2 k}(\nu) \varphi_{0}^{-}\right\rangle\right)
$$

Now, the result follows from (2.12), Lemma 2.1, and the fact that the coefficients of $\mathscr{O}_{\nu}$ are given by the scalar products $\left\langle P_{\nu} u_{\nu}^{ \pm}, u_{\nu}^{ \pm}\right\rangle$and $\left\langle P_{\nu} u_{\nu}^{ \pm}, u_{\nu}^{\mp}\right\rangle$, in which (2.15)(2.16) has to be inserted.

\section{Spectral Quantities of the Linear Problem}

Using Proposition 2.2, one can compute the two eigenvalues $\lambda_{\nu}^{ \pm}$of $P_{\nu} \Pi_{\nu}$. We find:

$$
\lambda_{\nu}^{ \pm} E_{0}+\sigma \pm \sqrt{\left|\varrho_{0} \cdot \nu+\beta_{2}\right|^{2}+(\mu+\delta)^{2}},
$$

where

$$
\begin{aligned}
\sigma & =\frac{1}{2}\left(\beta_{1}+\beta_{3}\right), \\
\delta & =\frac{1}{2}\left(\beta_{1}-\beta_{3}\right) .
\end{aligned}
$$

Denoting $\psi_{\nu}^{ \pm}$two associated eigenvectors normalized in $\mathscr{H}$, we have:

Proposition 3.1. In the situation of Proposition 2.2, and for $\nu$ satisfying $\left|\varrho_{0} . \nu+\beta_{2}\right|^{2}+$ $(\mu+\delta)^{2} \neq 0$, one has:

$$
\left\langle W \psi_{\nu}^{ \pm}, \psi_{\nu}^{ \pm}\right\rangle=F(\nu)+\frac{1}{\sqrt{\left|\varrho_{0} . \nu+\beta_{2}\right|^{2}+(\mu+\delta)^{2}}} G^{ \pm}(\nu),
$$

where $F$ and $G^{ \pm}$are odd analytic functions of $\nu$. Moreover, if $\varepsilon_{2}<1 / 2$, there exists a universal constant $C_{0}$ such that:

$$
\begin{gathered}
|F(\nu)| \leq C_{0}\|W\|\left(\frac{\pi \lambda+2 \mu}{\pi \lambda}\right)^{2} \varepsilon_{1} \\
\left|G^{ \pm}(\nu) \mp \operatorname{Re} \varrho_{0}\left(\varrho_{0} . \nu\right)\right| \leq C_{0}\|W\|\left(\frac{\pi \lambda+2 \mu}{\pi \lambda}\right)^{2}\left(\mu \varepsilon_{1}+\lambda_{1} \varepsilon_{1}^{3}\right) .
\end{gathered}
$$


Proof. Denote

$$
\psi_{\nu}^{ \pm}=a_{\nu}^{ \pm} u_{\nu}^{+}+b_{\nu}^{ \pm} u_{\nu}^{-}
$$

and

$$
\theta=\sqrt{\left|\varrho_{0} \cdot \nu+\beta_{2}\right|^{2}+(\mu+\delta)^{2}} .
$$

Then $a_{\nu}^{ \pm}$and $b_{\nu}^{ \pm}$satisfy $\left|a_{\nu}^{ \pm}\right|^{2}+\left|b_{\nu}^{ \pm}\right|^{2}=1$ and:

$$
\begin{aligned}
& (\theta \mp(\mu+\delta)) a_{\nu}^{ \pm}= \pm\left(\varrho_{0} \cdot \nu+\beta_{2}\right) b_{\nu}^{ \pm}, \\
& (\theta \pm(\mu+\delta)) b_{\nu}^{ \pm}= \pm\left(\overline{\varrho_{0}} \cdot \nu+\overline{\beta_{2}}\right) a_{\nu}^{ \pm} .
\end{aligned}
$$

\section{Moreover}

$$
\left\langle W \psi_{\nu}^{ \pm}, \psi_{\nu}^{ \pm}\right\rangle=\left|a_{\nu}^{ \pm}\right|^{2}\left\langle W u_{\nu}^{+}, u_{\nu}^{+}\right\rangle+\left|b_{\nu}^{ \pm}\right|^{2}\left\langle W u_{\nu}^{-}, u_{\nu}^{-}\right\rangle+2 \operatorname{Re} a_{\nu}^{ \pm} \overline{b_{\nu}^{ \pm}}\left\langle W u_{\nu}^{+}, u_{\nu}^{-}\right\rangle .
$$

Denoting $\varrho_{0}=\left(\varrho_{0,1}, \ldots, \varrho_{0, n}\right)$, we first prove:

Lemma 3.2. There exists a universal positive constant $C_{0}^{\prime}$ such that if $\varepsilon_{2}<1 / 2$ then:

$$
\begin{gathered}
\left|\left\langle W_{\jmath} u_{\nu}^{+}, u_{\nu}^{+}\right\rangle\right|+\left|\left\langle W_{j} u_{\nu}^{-}, u_{\nu}^{-}\right\rangle\right| \leq C_{0}^{\prime}\left\|W_{j}\right\|\left(\frac{\pi \lambda+2 \mu}{\pi \lambda}\right)^{2} \varepsilon_{1}, \\
\left|\left\langle W_{\jmath} u_{\nu}^{+}, u_{\nu}^{-}\right\rangle-\varrho_{0, \jmath}\right| \leq C_{0}^{\prime}\left\|W_{j}\right\|\left(\frac{\pi \lambda+2 \mu}{\pi \lambda}\right)^{2} e_{1}^{2}
\end{gathered}
$$

for all $j=1, \ldots, n$. Moreover, $\left\langle W_{j} u_{\nu}^{ \pm}, u_{\nu}^{ \pm}\right\rangle$are odd functions of $\nu$, while $\left\langle W_{\jmath} u_{\nu}^{+}, u_{\nu}^{-}\right\rangle$ is even.

Proof. By (2.12) (and since the transposed of $A_{\nu}$ is $\bar{A}_{\nu}$ ), one has:

$$
\begin{aligned}
& \left(\begin{array}{ll}
\left\langle W_{\jmath} u_{\nu}^{+}, u_{\nu}^{+}\right\rangle & \left\langle W_{j} u_{\nu}^{+}, u_{\nu}^{-}\right\rangle \\
\left\langle W_{j} u_{\nu}^{-}, u_{\nu}^{+}\right\rangle & \left\langle W_{\jmath} u_{\nu}^{-}, u_{\nu}^{-}\right\rangle
\end{array}\right) \\
& \quad=\left(\bar{A}_{\nu}\right)^{-1 / 2}\left(\begin{array}{cc}
\left\langle W_{\jmath} \varphi_{\nu}^{+}, \varphi_{\nu}^{+}\right\rangle & \left\langle W_{\jmath} \varphi_{\nu}^{+}, \varphi_{\nu}^{-}\right\rangle \\
\left\langle W_{j} \varphi_{\nu}^{-}, \varphi_{\nu}^{+}\right\rangle & \left\langle W_{j} \varphi_{\nu}^{-}, \varphi_{\nu}^{-}\right\rangle
\end{array}\right)\left(\bar{A}_{\nu}\right)^{-1 / 2} .
\end{aligned}
$$

Moreover, using (1.4) and (2.11) we see that $\left\langle W_{\jmath} \varphi_{\nu}^{ \pm}, \varphi_{\nu}^{ \pm}\right\rangle$are odd functions of $\nu$, while $\left\langle W_{\jmath} \varphi_{\nu}^{+}, \varphi_{\nu}^{-}\right\rangle$is even. As a consequence, according to (2.13)-(2.14), we get:

$$
\begin{aligned}
\left|\left\langle W_{\jmath} \varphi_{\nu}^{ \pm}, \varphi_{\nu}^{ \pm}\right\rangle\right| & =\left|\sum_{k+l \text { odd }}\left\langle W_{j} q_{k}(\nu) \varphi_{0}^{ \pm}, q_{l}(\nu) \varphi_{0}^{ \pm}\right\rangle\right| \\
& \leq\left\|W_{j}\right\|\left(\frac{\pi \lambda+2 \mu}{\pi \lambda}\right)^{2} \sum_{k+l \text { odd }} \varepsilon_{1}^{k+l} \\
& \leq C\left\|W_{j}\right\|\left(\frac{\pi \lambda+2 \mu}{\pi \lambda}\right)^{2} \varepsilon_{1}
\end{aligned}
$$

and

$$
\begin{aligned}
\left|\left\langle W_{j} \varphi_{\nu}^{+}, \varphi_{\nu}^{-}\right\rangle-\varrho_{0, j}\right| & =\left|\sum_{\substack{k+l \text { even } \\
k+l \geq 2}}\left\langle W_{\jmath} q_{k}(\nu) \varphi_{0}^{+}, q_{l}(\nu) \varphi_{0}^{-}\right\rangle\right| \\
& \leq C\left\|W_{\jmath}\right\|\left(\frac{\pi \lambda+2 \mu}{\pi \lambda}\right)^{2} \varepsilon_{1}^{2},
\end{aligned}
$$

where $C$ is some universal constant.

Then, the result follows from Lemma 2.1 by inserting (3.8)-(3.9) into (3.7). 
Going back to (3.5), we see that when $\theta \neq 0$ one can take one of the following two choices:

$$
a_{\nu}^{ \pm}=\frac{ \pm\left(\varrho_{0} . \nu+\beta_{2}\right)}{\sqrt{2 \theta}(\theta \mp(\mu+\delta))^{1 / 2}} ; \quad b_{\nu}^{ \pm}=\frac{(\theta \pm(\mu+\delta))^{1 / 2}}{\sqrt{2 \theta}}
$$

or

$$
a_{\nu}^{ \pm}=\frac{(\theta \pm(\mu+\delta))^{1 / 2}}{\sqrt{2 \theta}} ; \quad b_{\nu}^{ \pm}=\frac{ \pm\left(\overline{\varrho_{0}} \cdot \nu+\overline{\beta_{2}}\right)}{\sqrt{2 \theta}(\theta \pm(\mu+\delta))^{1 / 2}}
$$

depending on the sign of $\mu+\delta$ and the possible vanishing of $\varrho_{0} . \nu+\beta_{2}$.

Inserting e.g. (3.10) into (3.6), we get:

$$
\begin{aligned}
\left\langle W \psi_{\nu}^{ \pm}, \psi_{\nu}^{ \pm}\right\rangle= & \frac{\left|\varrho_{0} \cdot \nu+\beta_{2}\right|^{2}}{2 \theta(\theta \mp(\mu+\delta))}\left\langle W u_{\nu}^{+}, u_{\nu}^{+}\right\rangle+\frac{\theta \mp(\mu+\delta)}{2 \theta}\left\langle W u_{\nu}^{-}, u_{\nu}^{-}\right\rangle \\
& \pm 2 \operatorname{Re} \frac{\left(\varrho_{0} . \nu+\beta_{2}\right)}{2 \theta}\left\langle W u_{\nu}^{+}, u_{\nu}^{-}\right\rangle
\end{aligned}
$$

Then, noticing that

$$
\frac{\left|\varrho_{0} \cdot \nu+\beta_{2}\right|^{2}}{(\theta \mp(\mu+\delta))}=\theta \pm(\mu+\delta)
$$

we obtain the announced form for $\left\langle W \psi_{\nu}^{ \pm}, \psi_{\nu}^{ \pm}\right\rangle$, with

$$
\begin{aligned}
F(\nu) & =\frac{1}{2}\left(\left\langle W u_{\nu}^{+}, u_{\nu}^{+}\right\rangle+\left\langle W u_{\nu}^{-}, u_{\nu}^{-}\right\rangle\right), \\
G^{ \pm}(\nu) & = \pm \frac{\mu+\delta}{2}\left(\left\langle W u_{\nu}^{+}, u_{\nu}^{+}\right\rangle-\left\langle W u_{\nu}^{-}, u_{\nu}^{-}\right\rangle\right) \pm \operatorname{Re}\left(\varrho_{0} . \nu+\beta_{2}\right)\left\langle W u_{\nu}^{+}, u_{\nu}^{-}\right\rangle
\end{aligned}
$$

and actually, one can verify directly that the same formulas hold if we take the other choice $\left(3.10^{\prime}\right)$. Making use of Lemma 3.2 and the estimates in Proposition 2.2, the result follows.

\section{Symmetry Breaking - End of the Proof of Theorem 1.1}

Now, we turn back to the non-linear system (1.6), and we prove Theorem 1.1. Obviously, (1.6) is equivalent to:

$$
\left\{\begin{array}{l}
P_{\nu} \psi=E \psi \\
\nu=\tau\langle W \psi, \psi\rangle \\
\|\psi\|_{\mathscr{H}}=1
\end{array}\right.
$$

In particular, $\psi$ has to be an eigenvector of $P_{\nu}$ for some convenient value of $\nu$. When $\eta$ defined in (1.9) is sufficiently small (with a bound which is in principle explicit), it is clear that for $|\tau|<\tau_{0}$ the possible values of $\nu$ satisfying (4.1) verify all the conditions of Proposition 2.2.

We also have [with $\theta(\nu)$ defined in (3.4)]:

Lemma 4.1. Under assumptions (1.1)-(1.4) and (1.7), there exists a universal constant $\eta_{0}>0$ such that, if $\eta\left(\lambda, \mu, \varrho_{0},\|W\|, \tau_{0}\right) \leq \eta_{0}$, then any solution $\nu$ of (4.1) with $|\tau| \leq \tau_{0}$ satisfies:

$$
\theta(\nu) \neq 0 \text {. }
$$


Proof. Assume $\theta(\nu)=0$ and $\nu$ is a solution of (4.1). By the discussion of Sects. 2 and 3 (and in particular the fact that $\lambda_{\nu}^{+}=\lambda_{\nu}^{-}$in that case), we see that (4.1) is equivalent to the existence of $\alpha, \beta \in \mathbb{C}$ satisfying $|\alpha|^{2}+|\beta|^{2}=1$ and:

$$
\nu=\tau|\alpha|^{2}\left\langle W u_{\nu}^{+}, u_{\nu}^{+}\right\rangle+\tau|\beta|^{2}\left\langle W u_{\nu}^{-}, u_{\nu}^{-}\right\rangle+2 \tau \operatorname{Re} \alpha \bar{\beta}\left\langle W u_{\nu}^{+}, u_{\nu}^{-}\right\rangle .
$$

Using Lemma 3.2, (4.2) implies (with some other universal constant $C_{1}$ ):

$$
\left|\nu-2 \tau \varrho_{0} \operatorname{Re} \alpha \bar{\beta}\right| \leq C_{1}\|W\| \cdot|\tau| \varepsilon_{1},
$$

and therefore, by (2.8):

$$
\left|\varrho_{0} . \nu-2 \tau \varrho_{0}^{2} \operatorname{Re} \alpha \bar{\beta}\right| \leq C_{1}\left|\varrho_{0}\right| \frac{\|W\|^{2}}{\lambda}|\tau| .|\nu| .
$$

Also, $\theta(\nu)=0$ implies $\varrho_{0} . \nu+\beta_{2}(\nu)=0$ and thus, by Proposition 2.2:

$$
\left|\varrho_{0} . \nu\right| \leq C_{2} \lambda_{1} \varepsilon_{1}^{3}
$$

where, here and from now on, all the $C_{j}$ 's denote universal positive constants.

Since $\varepsilon_{1} \leq\|W\| \cdot|\nu| / \lambda$, and by (4.1) $|\nu| \leq\|W\| .|\tau|$, we get from (4.5):

$$
\left|\varrho_{0} . \nu\right| \leq C_{2} \frac{\lambda_{1}\|W\|^{5}}{\lambda^{3}}|\tau|^{2}|\nu|
$$

and thus, inserting (4.6) into (4.4) and using the fact that $\left|\varrho_{0}\right| \leq\|W\|$ :

$$
|\tau \operatorname{Re} \alpha \bar{\beta}| \leq \frac{C_{3}}{\left|\varrho_{0}\right|}\left(\left(\frac{\|W\|^{2}|\tau|}{\lambda}\right)^{2} \frac{\lambda_{1}\|W\|}{\lambda\left|\varrho_{0}\right|}+\frac{\|W\|^{2}|\tau|}{\lambda}\right)|\nu| \leq \frac{C_{3}}{\left|\varrho_{0}\right|}\left(\eta+\eta^{2}\right)|\nu| .
$$

We deduce from (4.3) and (4.7) that $\nu$ must satisfy:

$$
|\nu| \leq\left(C_{1} \frac{\|W\|^{2}}{\lambda} \tau_{0}+2 C_{3}\left(\eta+\eta^{2}\right)\right)|\nu| \leq C_{4}\left(\eta+\eta^{2}\right)|\nu| .
$$

Therefore, $\nu$ has to be zero if we take $\eta+\eta^{2}<1 / C_{4}$. But $\theta(0)=\mu>0$ and this is in contradiction with the assumption that $\theta(\nu)=0$.

Now, from Lemma 4.1 and Proposition 3.1, we see that the solutions $\nu$ of (4.1) must satisfy one of the two following equations:

$$
\theta(\nu) \nu=\tau \theta(\nu) F(\nu)+\tau G^{ \pm}(\nu)
$$

Conversely, any solution $\nu$ of (4.9) $)_{ \pm}$gives rise to the solutions $\psi=\psi_{\nu}^{ \pm}$and $\psi=S \psi_{\nu}^{ \pm}$ of (4.1) associated to the same energy $E=\lambda_{\nu}^{ \pm}$.

Since $F$ and $G^{ \pm}$are odd, $\nu=0$ is always a solution of $(4.9)_{ \pm}$, and it corresponds precisely to the symmetrical solutions $\varphi_{0}^{ \pm}$of (1.6).

From now on, we assume $\nu \neq 0$. First looking at $\theta$ as an extra-parameter, we consider the equation:

$$
\theta \nu=\tau \theta F(\nu)+\tau G^{ \pm}(\nu)
$$


and we solve it by the use of polar coordinates:

$$
\nu=t \omega ; \quad t>0 ; \quad \omega \in \mathbb{S}^{n-1}
$$

From Proposition 3.1 and its proof, we see that $F$ and $G^{ \pm}$can be written:

$$
\begin{aligned}
F(\nu) & =\sum_{|\alpha| \text { odd }} f_{\alpha} \nu^{\alpha}, \\
G^{ \pm}(\nu) & =\sum_{|\alpha| \text { odd }} g_{\alpha}^{ \pm} \nu^{\alpha}
\end{aligned}
$$

with, for all $\alpha \in \mathbb{N},|\alpha|$ odd:

$$
\begin{aligned}
& \left|f_{\alpha}\right| \leq C_{5}^{|\alpha|+1} \frac{\|W\|^{|\alpha|+1}}{\lambda^{|\alpha|}} \\
& \left|g_{\alpha}^{ \pm}\right| \leq \lambda_{1} C_{5}^{|\alpha|+1} \frac{\|W\|^{|\alpha|+1}}{\lambda^{|\alpha|}}
\end{aligned}
$$

and

$$
\left|\sum_{|\alpha|=1} g_{\alpha}^{ \pm} \nu^{\alpha} \mp\left(\varrho_{0} . \nu\right) \varrho_{0}\right| \leq C_{5} \frac{\|W\|^{2}}{\lambda} \mu|\nu| \text {. }
$$

We denote $F_{0} \cdot \nu:=\sum_{|\alpha|=1} f_{\alpha} \nu^{\alpha}$ and $G_{0}^{ \pm} \cdot \nu:=\sum_{|\alpha|=1} g_{\alpha}^{ \pm} \nu^{\alpha}$.

Substituting (4.11) into (4.10) \pm and dividing by $\tau t$, we get:

$$
\left(G_{0}^{ \pm}+\theta F_{0}-\frac{\theta}{\tau}\right) \cdot \omega+\sum_{\substack{|\alpha| \geq 3 \\|\alpha| \text { odd }}}\left(g_{\alpha}^{ \pm}+\theta f_{\alpha}\right) t^{|\alpha|-1} \omega^{\alpha}=0
$$

For $t=0,(4.15)_{ \pm}$becomes

$$
\left(G_{0}^{ \pm}+\theta F_{0}\right) \cdot \omega=\frac{\theta}{\tau} \omega
$$

Since the eigenvalues of the linear map $\nu \mapsto\left(\varrho_{0} . \nu\right) \varrho_{0}$ are $\varrho_{0}^{2}$ (multiplicity 1) and 0 (multiplicity $n-1$ ), we deduce from (4.13)-(4.14) that the eigenvalues $\lambda_{1}^{ \pm}(\theta), \ldots, \lambda_{n}^{ \pm}(\theta)$ of $G_{0}^{ \pm}+\theta F_{0}$ satisfy:

$$
\begin{aligned}
\left|\lambda_{1}^{ \pm}(\theta) \mp \varrho_{0}^{2}\right| & \leq C_{5} \frac{\|W\|^{2}}{\lambda}(\mu+|\theta|), \\
\left|\lambda_{j}^{ \pm}(\theta)\right| & \leq C_{5} \frac{\|W\|^{2}}{\lambda}(\mu+|\theta|) \text { for } j \geq 2, \\
\left|\frac{\partial}{\partial \theta} \lambda_{j}^{ \pm}(\theta)\right| & \leq C_{5} \frac{\|W\|^{2}}{\lambda} \text { for } 1 \leq j \leq n .
\end{aligned}
$$
Therefore, we see by the implicit function theorem that if $\tau_{0} \frac{\|W\|^{2}}{\lambda}<\frac{1}{C_{5}}$, then for
any $j$, the equation:

$$
\frac{\theta}{\tau}=\lambda_{j}^{ \pm}(\theta)
$$


admits a unique solution $\theta_{j}^{ \pm}=\theta_{j}^{ \pm}(\tau)$ for any $\tau$ satisfying $0<|\tau|<\tau_{0}$. As a consequence of (4.17), these $\theta_{j}^{ \pm}$'s satisfy:

$$
\begin{aligned}
\left|\theta_{1}^{ \pm} \mp \tau \varrho_{0}^{2}\right| & \leq C_{5}|\tau| \frac{\|W\|^{2}}{\lambda}\left(\mu+\left|\theta_{1}^{ \pm}\right|\right), \\
\left|\theta_{\jmath}^{ \pm}\right| & \leq C_{5}|\tau| \frac{\|W\|^{2}}{\lambda}\left(\mu+\left|\theta_{j}^{ \pm}\right|\right) \quad(j \geq 2) .
\end{aligned}
$$

Moreover, the eigenvector $\omega_{1}^{ \pm} \in \mathbb{S}^{n-1}$ associated to $\theta_{1}^{ \pm}$verifies by the same procedure (and up to a factor \pm 1 ):

$$
\left|\omega_{1}^{ \pm}-\frac{\varrho_{0}}{\left|\varrho_{0}\right|}\right| \leq C_{5}\left|\theta_{1}^{ \pm}\right| \frac{\|W\|^{2}}{\lambda}
$$

Now for $t \neq 0$, we see that any solution $(\omega, \theta)$ of $(4.15)_{ \pm}$must satisfy for some $j \in\{1, \ldots, n\}$ :

$$
\left|\lambda_{j}^{ \pm}(\theta)-\frac{\theta}{\tau}\right| \leq C_{6} \frac{\|W\|^{4}}{\lambda^{3}}\left(\lambda_{1}+|\theta|\right) t^{2},
$$

and therefore, by (4.17):

$$
\left(\frac{1}{|\tau|}-C_{5} \frac{\|W\|^{2}}{\lambda}\right)\left|\theta-\theta_{j}^{ \pm}\right| \leq C_{6} \frac{\|W\|^{4}}{\lambda^{3}}\left(\lambda_{1}+|\theta|\right) t^{2} .
$$

Assuming e.g. $C_{5} \eta_{0} \leq 1 / 2$, this gives for $|t| \leq|\tau| .\|W\|$ :

$$
\left|\theta-\theta_{j}^{ \pm}\right| \leq C_{7}|\tau| \frac{\|W\|^{4}}{\lambda^{3}}\left(\lambda_{1}+|\theta|\right) t^{2} \leq C_{7}|\tau| \frac{\|W\|^{2}}{\lambda}\left(\frac{\lambda_{1}\|W\|^{2}}{\lambda^{2}} t^{2}+\eta^{2}|\theta|\right),
$$

and thus, by (4.18):

$$
\begin{gathered}
\left|\theta \mp \tau \varrho_{0}^{2}\right| \leq C_{8}|\tau| \frac{\|W\|^{2}}{\lambda}\left(\mu+\frac{\lambda_{1}\|W\|^{2}}{\lambda^{2}} t^{2}+|\theta|\right) \quad \text { if } \quad j=1 \\
|\theta| \leq C_{8}|\tau| \frac{\|W\|^{2}}{\lambda}\left(\mu+\frac{\lambda_{1}\|W\|^{2}}{\lambda^{2}} t^{2}\right) \quad \text { if } \quad j \geq 2 .
\end{gathered}
$$

Now, we have the following improvement of Lemma 4.1:

Lemma 4.2. If $\eta_{0}$ is taken sufficiently small, and $\eta\left(\lambda, \mu, \varrho_{0},\|W\|, \tau_{0}\right) \leq \eta_{0}$, then any solution $\nu$ of (4.1) with $|\tau| \leq \tau_{0}$ satisfies:

$$
\theta(\nu) \geq 2 C_{8} \tau_{0} \frac{\|W\|^{2}}{\lambda}\left(\mu+\frac{\lambda_{1}\|W\|^{2}}{\lambda^{2}}|\nu|^{2}\right)
$$

Proof. We adopt the same strategy as for Lemma 4.1, except that now $(\alpha, \beta)$ is forced to be $\left(a_{\nu}^{ \pm}, b_{\nu}^{ \pm}\right)$since we already know that $\theta(\nu) \neq 0$. But this changes nothing in 
(4.3)-(4.4) and, assuming $\theta(\nu) \leq 2 C_{8} \tau_{0} \frac{\|W\|^{2}}{\lambda}\left(\mu+\frac{\lambda_{1}\|W\|^{2}}{\lambda^{2}}|\nu|^{2}\right)$, (4.6) must be
modified into:

$$
\left|\varrho_{0} . \nu\right| \leq C_{2} \frac{\lambda_{1}\|W\|^{5}}{\lambda^{3}} \tau_{0}^{2}|\nu|+2 C_{8} \tau_{0} \frac{\|W\|^{2}}{\lambda}\left(\mu+\frac{\lambda_{1}\|W\|^{2}}{\lambda^{2}}|\nu|^{2}\right) .
$$

As for (4.8) this gives for $\eta \leq 1$ :

$$
\begin{aligned}
|\nu| & \leq C_{4}\left(\eta+\eta^{2}\right)|\nu|+2 C_{8} \tau_{0} \frac{\|W\|^{2}}{\lambda\left|\varrho_{0}\right|}\left(\mu+\frac{\lambda_{1}\|W\|^{2}}{\lambda^{2}}|\nu|^{2}\right) \\
& \leq 2\left(C_{4}+C_{8}\right) \eta|\nu|+2 C_{8} \frac{\|W\|^{2}}{\lambda\left|\varrho_{0}\right|} \tau_{0} \mu,
\end{aligned}
$$

and thus, if $2\left(C_{4}+C_{8}\right) \eta_{0} \leq 1 / 2$ :

$$
|\nu| \leq 4 C_{8} \frac{\|W\|^{2}}{\lambda\left|\varrho_{0}\right|} \tau_{0} \mu
$$

But then, using (3.2), (2.8) and Proposition 2.2:

$$
|\delta(\nu)| \leq C_{9} \frac{\lambda_{1}\|W\|^{6}}{\lambda^{4} \varrho_{0}^{2}} \tau_{0}^{2} \mu^{2},
$$

i.e.

$$
|\delta(\nu)| \leq C_{9}\left(\frac{\tau_{0}\|W\|^{2}}{\lambda}\right)^{2} \frac{\mu \lambda_{1}\|W\|^{2}}{\lambda^{2}\left|\varrho_{0}\right|^{2}} \mu \leq C_{9} \eta^{3} \mu .
$$

As a consequence

$$
\theta(\nu) \geq \mu+\delta(\nu) \geq\left(1-C_{9} \eta^{3}\right) \mu
$$

Since also

$$
\theta(\nu) \leq 2 C_{8} \eta\left(\mu+\frac{\lambda_{1}\|W\|^{2}}{\lambda^{2}}|\nu|^{2}\right) \leq 2 C_{8} \eta\left(1+16 C_{8}^{2} \eta^{3}\right) \mu,
$$

the contradiction is immediate for $\eta$ small enough.

As a consequence of Lemma 4.2 and (4.21), we see that we only have to investigate the possible solutions of (4.15) \pm which satisfy (4.20) with $j=1$.

Denoting $A^{ \pm}(t, \omega, \theta)$ the left-hand side of $(4.15)_{ \pm}$, and computing its tangential gradient $\partial_{\omega} A^{ \pm}$with respect to $\omega$ on $\mathbb{S}^{n-1}$, we find, using $(4.20)_{j=1}$ :

$$
\begin{aligned}
\left\|\partial_{\omega} A^{ \pm}-\left(G_{0}^{ \pm}+\theta_{1}^{ \pm} F_{0}-\frac{\theta_{1}^{ \pm}}{\tau}\right)\right\| & \leq C_{10} \frac{\|W\|^{4}}{\lambda^{3}}\left(\lambda_{1}+|\tau| \cdot\|W\|^{2}\right) t^{2} \\
& \leq C_{10} \varrho_{0}^{2}\left(\eta^{2}+\eta^{3}\right)\left(\frac{t}{|\tau| \cdot \mid\|W\|}\right)^{2} .
\end{aligned}
$$

Denoting $\left\langle\omega_{1}^{ \pm}\right\rangle^{\perp}$ the subspace of $\mathbb{R}^{n}$ orthogonal to $\omega_{1}^{ \pm}$, we also see by using (4.17):

$$
\left\|\left.\left(G_{0}^{ \pm}+\theta_{1}^{ \pm} F_{0}-\frac{\theta_{1}^{ \pm}}{\tau}\right)\right|_{\left\langle\omega_{1}^{ \pm}\right\rangle^{\perp}}\right\| \geq \frac{1-C_{5} \eta}{|\tau|} \operatorname{Min}_{j \geq 2}\left|\theta_{1}^{ \pm}-\theta_{j}^{ \pm}\right|
$$


and therefore, by (4.18):

$$
\left\|\left.\left(G_{0}^{ \pm}+\theta_{1}^{ \pm} F_{0}-\frac{\theta_{1}^{ \pm}}{\tau}\right)\right|_{\left\langle\omega_{1}^{ \pm}\right\rangle \perp}\right\| \geq \varrho_{0}^{2}\left(1-C_{11} \eta\right) .
$$

Possibly by taking $\eta_{0}$ smaller, we deduce from (4.22)-(4.23) that $\partial_{\omega} A^{ \pm}(t, \omega, \theta)$ has rank $n-1$ for $|t| \leq\|W\| \cdot|\tau|$ and $\theta$ satisfying (4.20) $)_{j=1}$. Since also $\partial_{\theta} A^{ \pm}$does not vanish there (and is actually close to $-\omega / \tau$ ), we conclude that for all $|t| \leq\|W\| \cdot|\tau|$, $(4.15)_{ \pm}$admits a unique solution $\left(\tilde{\omega}_{1}^{ \pm}(t), \tilde{\theta}_{1}^{ \pm}(t)\right)$ verifying $(4.20)_{j=1}$. Moreover, $\tilde{\omega}_{1}^{ \pm}(t)$ and $\tilde{\theta}_{1}^{ \pm}(t)$ depend analytically on $t^{2}$ and satisfy:

$$
\begin{aligned}
& \tilde{\omega}_{1}^{ \pm}(t)=\omega_{1}^{ \pm}+\sum_{k \geq 1} a_{k}^{ \pm} t^{2 k} \\
& \tilde{\theta}_{1}^{ \pm}(t)=\theta_{1}^{ \pm}+\sum_{k \geq 1} b_{k}^{ \pm} t^{2 k}
\end{aligned}
$$

with

$$
\begin{aligned}
& \left|a_{k}^{ \pm}\right| \leq C_{12}^{k+1} \frac{\|W\|^{2 k}}{\lambda^{2 k}}, \\
& \left|b_{k}^{ \pm}\right| \leq C_{12}^{k+1} \frac{\|W\|^{2 k}}{\lambda^{2 k}}|\tau| .
\end{aligned}
$$

Now, to obtain solutions for (4.9) (and then finish the proof of Theorem 1.1), it remains to solve the two following equations where $t$ is the unknown (and $\theta=\theta(\nu$ ) is again the function defined in (3.4)):

$$
\tilde{\theta}_{1}^{ \pm}(t)=\theta\left(t \tilde{\omega}_{1}^{ \pm}(t)\right)
$$

which are equivalent to:

$$
\left\{\begin{array}{l}
\left|\varrho_{0} \cdot t \tilde{\omega}_{1}^{ \pm}(t)+\beta_{2}\left(t \tilde{\omega}_{1}^{ \pm}(t)\right)\right|^{2}+\left(\mu+\delta\left(t \tilde{\omega}_{1}^{ \pm}(t)\right)\right)^{2}=\tilde{\theta}_{1}^{ \pm}(t)^{2} \\
\tilde{\theta}_{1}^{ \pm}(t) \geq 0
\end{array}\right.
$$

In view of (4.18) and (4.24), it is easily seen that only at most one of the two systems $(4.27)_{ \pm}$can admit a solution, depending on the sign of $\tau$. Assume for instance $\tau \leq 0$ (which actually corresponds to the physical case), then only $\tilde{\theta}_{1}^{-}(t)$ is non-negative for $t$ small enough, and the first equation of (4.27)_ can be re-written:

$$
\left(\theta_{1}^{-}\right)^{2}-\mu^{2}=t^{2}\left(\left(\varrho_{0} \cdot \omega_{1}^{-}\right)^{2}+\sum_{k \geq 1} c_{k} t^{2 k}\right)
$$

with some coefficients $c_{k}$ satisfying estimates analogous to (4.25). Using also (4.19), we see that

$$
\left(\varrho_{0} \cdot \omega_{1}^{-}\right)^{2}+\sum_{k \geq 1} c_{k} t^{2 k} \geq\left|\varrho_{0}\right|^{2}\left(1-C_{13} \eta\right) .
$$

This proves that (4.28) admit a solution if and only if $\theta_{1}^{-} \geq \mu$, and by (4.18) this is equivalent to:

$$
\tau \leq \tau_{-}
$$


where $\tau_{-} \leq 0$ satisfies:

$$
\left|\tau_{-}+\frac{\mu}{\varrho_{0}^{2}}\right| \leq C_{14} \eta \frac{\mu}{\varrho_{0}^{2}}
$$

An analogous result can be obtained in the same way for $\tau \geq 0$, and this proves part (i) of Theorem 1.1, as well as the existence of the $\tilde{\varphi}_{\tau}^{ \pm}$'s in parts (ii) and (iii). Their corresponding energies are given by:

$$
E_{\tau}^{ \pm}=\lambda_{\nu_{ \pm}}^{ \pm}
$$

where $\nu_{ \pm}=t_{ \pm} \tilde{\omega}_{1}^{ \pm}\left(t_{ \pm}\right), t_{ \pm}$being the non-negative solution of $(4.27)_{ \pm}$when $\pm \tau \geq \pm \tau_{ \pm}$. By the estimates (4.29) and (4.18)-(4.19), we get:

$$
t_{ \pm}=\left|\varrho_{0}\right| \sqrt{\tau^{2}-\tau_{ \pm}^{2}}(1+\odot(\eta))
$$

where the $\mathscr{Q}(\eta)$ is uniform with respect to all the parameters as long as $\eta \leq \eta_{0}$. Therefore

$$
\begin{gathered}
\nu_{ \pm}=\varrho_{0} \sqrt{\tau^{2}-\tau_{ \pm}^{2}}(1+\mathcal{O}(\eta)), \\
\varrho_{0} \cdot \nu_{ \pm}=\left|\varrho_{0}\right|^{2} \sqrt{\tau^{2}-\tau_{ \pm}^{2}}(1+\mathcal{O}(\eta)),
\end{gathered}
$$

which, once inserted into (3.1), gives:

$$
E_{\tau}^{ \pm}=E_{0} \pm\left|\varrho_{0}\right|^{2} \sqrt{\tau^{2}-\tau_{ \pm}^{2}+\frac{\mu^{2}}{\left|\varrho_{0}\right|^{4}}}(1+\mathscr{\sigma}(\eta))
$$

i.e. (using (4.30) and the analogous estimate for $\tau_{+}$):

$$
E_{\tau}^{ \pm}=E_{0} \pm|\tau| \varrho_{0}^{2}(1+\mathscr{\odot}(\eta))
$$

It remains to compute $\left\langle W \tilde{\varphi}_{\tau}^{ \pm}, \tilde{\varphi}_{\tau}^{ \pm}\right\rangle=\left\langle W \psi_{\nu_{ \pm}^{ \pm}}^{ \pm}, \psi_{\nu_{ \pm}}^{ \pm}\right\rangle$. Using Proposition 3.1 and (4.32) we find:

$$
\left|\left\langle W \tilde{\varphi}_{\tau}^{ \pm}, \tilde{\varphi}_{\tau}^{ \pm}\right\rangle \mp \frac{\sqrt{\tau^{2}-\tau_{ \pm}^{2}}}{|\tau|} \varrho_{0}\right| \leq C_{15} \eta\left|\varrho_{0}\right| \frac{\sqrt{\tau^{2}-\tau_{ \pm}^{2}}}{|\tau|}
$$

This finishes the proof of Theorem 1.1.

Acknowledgements. This work was partially supported by the Italian MURST. We would like to thank Ch. Chardonnet, from the "Laboratoire de Physique des Lasers" of Université Paris-Nord, for several useful discussions concerning the physical aspects of the problem. We also thank G. Jona Lasinio for the interest he has shown in our work, and for giving us many relevant references. 


\section{References}

[An] Anderson, P.W.: More is different. Science (N.Y.) 177, 393 (1972)

[ClJo1] Claverie, P., Jona Lasinio, G.: The instability of tunneling and the concept of molecule structure in quantum mechanics: the case of pyramidal molecules and the enantiomer problem. Phys. Rev. A 33, 2245-2253 (1986)

[ClJo2] Claverie, P., Jona Lasinio, G.: Symmetry breaking and classical behavior. Progress of Theoretical Physics Supplement No. 86, 54-59 (1986)

[Da] Davies, E.B.: Symmetry breaking for a non-linear Schrödinger equation. Commun. Math. Phys. 64, 191-210 (1979)

[Ha] Hand, F.: Zeits für Phys. 45, 805 (1927)

[HeSj] Helffer, B., Sjöstrand, J.: Multiple wells in the semiclassical limit. I. Comm. P.D.E. 9(4), $337-408$ (1984)

[KMSW] Klein, M., Martinez, A., Seiler, R., Wang, X.P.: On the Born-Oppenheimer expansion for polyatomic molecules. Commun. Math. Phys. 143, 607-639 (1992)

[Ma] Margenau, H.: Inversion frequency of ammonia and molecular interaction. Phys. Rev. 75, 1450 (1949)

[Pf] Pfeifer, P.: A nonlinear Schrödinger equation yielding the "Shape of molecules" by symmetry breaking. Quantum mechanics in mathematics, N.Y., Chemistry and physics. K.E. Gustafson, W.P. Reinhardt (eds.) 1981

[Pr] Prat, R.F.: Spontaneous deformation of hydrogen atom shape in an isotropic environment. J. Phys. France 49, 635-641 (1988)

[Si] Simon, B.: Semiclassical limit of low lying eigenvalues. I. Ann. Inst. H. Poincaré, 38, 295-307 (1983)

[Wi] Wightman, A.S.: Some comments on the quantum theory of measurement. Probabilistic methods in mathematical Physics. Eds. F. Guerra, M. Loffredo, C. Marchioro. Singapore: World Scientific 1992, pp. 411-438

[WiGl] Wightman, A.S., Glance, N.: Superselection rules in molecules. Nucl. Phys. B (Proc. Suppl.) i, 202-206 (1989)

Communicated by B. Simon 